\title{
A Quantitative Image-Based in vitro Assay for Induction of Phospholipidosis in Hepatocytes
}

\author{
G.D. Gagne, R.J. Gum, R.A. Jolly, M. A. Heindel and J. A. Fagerland
}

Abbott Laboratories, 200 Abbott Park Road, Abbott Park, IL 60064

Phospholipidosis (PL) is a lipid storage disorder in which phospholipids accumulate in lysosomes as lamellar bodies [1]. Cationic amphiphilic drugs may induce PL in vivo, which is often associated with undesirable drug accumulation and associated toxicities in affected tissues. Identification of PL-inducing compounds early in drug development allows elimination of less desirable compounds so that resources are available for more promising drug candidates.

Induction of PL can be studied in vitro by incubating cultured cells with NBD-PE, a fluorescent analog of phosphatidylethanolamine [2]. Cells exhibiting PL are identified by the accumulation of fluorescent granules in their cytoplasm. We have used this technique to evaluate the effects of drug compounds and their metabolites in primary cultures of rat and human hepatocytes [3]. Quantitating this assay by methods such as fluorimetry is difficult because the lipid probe also accumulates in the cytoplasm of dead cells, which are always present in primary cultures. We have developed an image-based assay to overcome these difficulties.

The assay was validated using amiodarone, a known inducer of PL, as a positive control. Primary rat hepatocytes incubated for 24 hours with amiodarone and NBD-PE showed an accumulation of fluorescent granules in the cytoplasm, which was more pronounced at higher concentrations of drug (Fig. 1). For each field examined, a fluorescence image and a brightfield image (for determination of total cell area) were acquired. Image analysis was used to measure the area occupied by fluorescent granules as a percentage of the total cell area, and this percentage was used as an indicator of PL. The results showed a clear dose response (Fig. 2). The decrease in the area occupied by fluorescent granules at $25 \mu \mathrm{M}$ was likely due to toxicity of the drug at high concentrations.

Reversibility of PL is important for determining the safety of compounds producing this effect [3]. Using amiodarone, we examined the reversibility of PL after withdrawal of the drug by measuring fluorescence at time points from 1 to 48 hours after washing out the drug. At low drug concentrations, PL was almost completely reversed by 24 hours, but cells treated with the highest concentration maintained near-peak levels up to 32 hours after drug removal (Fig. 3).

We used the assay to examine induction of PL in cultured primary human hepatocytes exposed to three structurally similar compounds being evaluated for the same therapeutic indication. One of the three compounds showed a significant dose-related induction of PL (Figs. 4, 5), whereas the other two were similar to vehicle controls at all tested concentrations. This information was one of the criteria used to select compounds for further development.

References

[1] M.J. Reasor, Toxicol. Appl. Pharmacol. 97 (1989) 47.

[2] R.G. Ulrich et al., Toxicol. Meth. 1 (1991) 89.

[3] R.J. Gum et al., Biochem. Pharmacol. 62 (2001) 1661. 

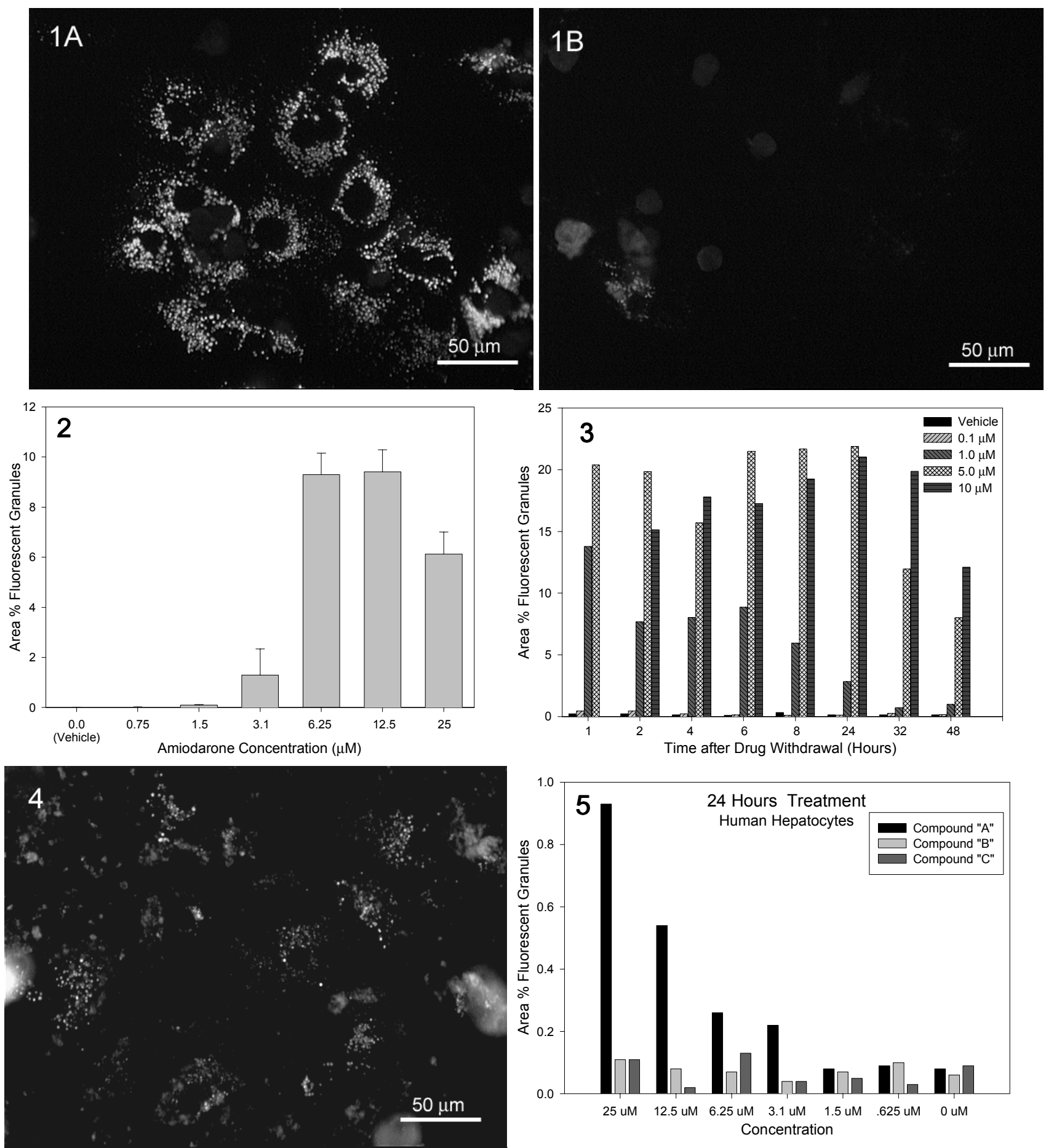

FIG. 1. Cultured primary rat hepatocytes treated for 24 hours with $6.25 \mu \mathrm{M}$ (A) and $0.75 \mu \mathrm{M}$ (B) amiodarone. FIG. 2. Dose-response of phospholipidosis induction in primary rat hepatocytes treated with amiodarone. FIG. 3. Persistence of phospholipidosis in rat hepatocytes after removal of amiodarone from the culture medium. FIG. 4. Cultured primary human hepatocytes incubated for 24 hours with $12.5 \mu \mathrm{M}$ of a compound (Compound "A") which induced phospholipidosis. FIG. 5. Comparison of phospholipidosis induction in primary human hepatocytes by three related compounds. 